\title{
Reconfigurable dual band microstrip patch antenna for Software Defined Radio applications
}

\author{
Ibrahim Tekin ${ }^{1}$, Michael Knox ${ }^{2}$ \\ ${ }^{1}$ Electronics Engineering, Sabanci University \\ 34956, Istanbul, Turkey \\ ${ }^{2}$ Electrical Engineering, Polytechnic Institute of NYU \\ Brooklyn, NY, USA \\ E-mail: tekin@sabanciuniv.edu
}

In this paper, a reconfigurable microstrip patch antenna with RF pin diode switches is implemented for dual band of $2.4 \mathrm{GHz}$ and $5.6 \mathrm{GHz}$ Software Defined Radio (SDR) applications. For the dual band SDR system, the use of a single antenna with a wide bandwidth to cover both of the bands can be limiting for low power level signal applications due to wideband noise. A reconfigurable nested microstrip patch antenna is designed on a Rogers 5880 RT/DUROID substrate which is fed by a coaxial probe from the back side of the grounded substrate. RF switching circuitry involves four RF pin diodes at each side of the inner patch. The dual bands of $2.4 \mathrm{GHz}$ and $5.6 \mathrm{GHz}$ frequency operation can be simply obtained by switching the PIN diodes on and off. The antenna is well matched and achieves approximately $7 \mathrm{dBi}$ simulated gain at both frequency bands. Simulation results show that the nested patch antenna is suitable for dual band SDR applications.

\section{Introduction}

In this paper, the design of a reconfigurable microstrip patch antenna with RF pin diode switches is presented for SDR applications. There have been many examples of antennas for SDR and frequency reconfigurable antennas for different applications [1-2]. However, these antennas are optimized for one of the frequency bands and may require additional reconfigurable tuning circuits for good impedance matching [3]. We propose a reconfigurable microstrip patch antenna with no additional matching circuits for WLAN systems at $2.4 \mathrm{GHz}$ and $5.6 \mathrm{GHz} .2 .4 \mathrm{GHz}$ band is commonly used by many WLAN systems. However, with many deployed systems at $2.4 \mathrm{GHz}$ band, RF interference and noise level can be much higher at this band. There is also $5.6 \mathrm{GHz}$ band available for WLAN and in general, use of the systems at this band is not that widespread as the use of $2.4 \mathrm{GHz}$ systems. The proposed reconfigurable antenna paves the way for an SDR or cognitive radio application where both frequency bands can be chosen to operate depending on the interference level for WLAN.

\section{Reconfigurable microstrip patch antenna}

A schematic view of the proposed antenna is plotted in Figure 1. The antenna has two microstrip nested patches with sizes L1, W1 designed to operate at $2.4 \mathrm{GHz}$, and L2, W2 designed to operate at 5.6 GHz. The nested patches are connected or disconnected electrically using RF pin diodes located on each side of the inner patch. To complete the return of the RF PIN diodes current, a grounding return is supplied on the outer patch corners using four quarter wavelength shorted stubs which are short circuits for DC bias and open circuits at $2.4 \mathrm{GHz}$. The patch antennas are connected from one point with a probe which is also optimized for both of the patches such that a good impedance matching is obtained. By the nested patch design, one can 
obtain a good impedance match for any two different frequencies by adjusting the relative position of one microstrip patch with respect to the other patch. DC bias for all RF pin diodes are given from one supply with a bias tee connected at the input port of the antenna. If the antenna is to operate at $5.6 \mathrm{GHz}$, the RF pin diodes will be reverse-biased so that the inner and outer patches are disconnected. Note that reverse bias capacitance of the pin diodes may change the resonance frequency of the inner patch. For an operation at $2.4 \mathrm{GHz}$, the pin diodes will be turned on and the inner and outer patches will be connected to each other electrically and again note that the series resistance of the pin diodes may degrade the antenna gain at this frequency.

One of the features of this antenna is that by changing the relative position of the patches, a good impedance matching and radiation pattern can be obtained for both of the frequencies 2.4/5.6 $\mathrm{GHz}$. In the design procedure, first, the inner patch antenna at $5.6 \mathrm{GHz}$ is designed by finding its probe feed location for a good impedance matching. This can be done easily by commercially available EM software such as ADS Momentum. Once, a good matching is obtained for the inner patch, a good matching impedance can be obtained for the outer patch that will work at $2.4 \mathrm{GHz}$ by moving the inner patch position with the feed point fixed with respect to the outer patch in $x-y$ directions as shown in Figure 1. With nested patch antenna, a good impedance matching and good radiation pattern can be obtained for both of the two frequencies which may not be available for dual band fixed antenna designs.

\section{Measurement and Simulation results}

The reconfigurable dual band nested microstrip patch antenna is designed on an Rogers 5880 RT/DUROID substrate with a relative dielectric constant of $\varepsilon_{r}=2.2$ and with a thickness of $0.78 \mathrm{~mm}$. The antenna is fed by a coaxial probe from the back side of the grounded substrate. RF switching circuitry involves four RF pin diodes at each side of the inner patch that require three volts for biasing. The dual bands of $2.4 \mathrm{GHz}$ and $5.6 \mathrm{GHz}$ frequency operation can be simply obtained by switching the PIN diodes on and off. The antenna is simulated using ADS Momentum tool. The fabricated microstrip patch antenna is shown in Figure 2. The RF pin diodes are MMP7000 series from Aeroflex corporation, which requires around $3 \mathrm{~mA}$ of forward current for bias per diode. The antenna dimensions for $2.4 / 5.6 \mathrm{GHz}$ operations are optimized using ADS momentum with following dimensions: the inner patch dimensions are $\mathrm{W} 1=15.9$ $\mathrm{mm}$ and $\mathrm{L} 1=17.3 \mathrm{~mm}$. Outer patch dimensions are $\mathrm{W} 2=39.8 \mathrm{~mm}$ and $\mathrm{L} 2=41.3 \mathrm{~mm}$. The short circuited stub width is $2 \mathrm{~mm}$ and the length is $\mathrm{D} 1=21.1 \mathrm{~mm}$. The gap between inner and outer patch is $1.1 \mathrm{~mm}$. The coaxial probe is connected to a point which is $6.7 \mathrm{~mm}$ from the left side of the inner patch and $7.1 \mathrm{~mm}$ from the bottom edge of the inner patch. Simulated and measured input return losses (S11), simulated radiation patterns (co and cross polarizations) of the nested patch antenna are given in Figures 3 and 4 for both frequencies. In Figure 3.a, the simulated and measured return losses of the antenna are shown for $2-7 \mathrm{GHz}$ band. The simulation and measurement results are in good agreement, and for the $2.4 \mathrm{GHz}$ band, the antenna achieves a $10 \mathrm{~dB}$ measured bandwidth of $15 \mathrm{MHz}$. Also, notice in the Figure 3.a that higher order modes of the patch antenna are also excited at higher frequency band $(4-7 \mathrm{GHz})$ for the $2.4 \mathrm{GHz}$ band mode, however, these higher order modes can be easily filtered out. The radiation patterns are simulated at $2.337 \mathrm{GHz}$ (frequency of smallest return loss of $-25 \mathrm{~dB}$ ). In Figure 4.a, the simulated and measured return losses of the antenna are shown for the band of $5.6 \mathrm{GHz}$. The antenna achieves a $-10 \mathrm{~dB}$ measured bandwidth of $90 \mathrm{MHz}$. The minimum of $\mathrm{S} 11$ is measured to be $-22 \mathrm{~dB}$ at $5.54 \mathrm{GHz}$, achieving a value of $-16 \mathrm{~dB}$ at $5.6 \mathrm{GHz}$ where the radiation patterns are 
simulated for the antenna. In Figures 3.b and 4.b, simulated radiation patterns of the antenna are shown for E plane (phi = 0), for $2.337 \mathrm{GHz}$ and 5.6 GHz. The antenna is linearly polarized for both frequencies and achieves co/cross polar ratio of $20.5 \mathrm{~dB}$ at $2.337 \mathrm{GHz}$ and almost $44 \mathrm{~dB}$ at 5.6 GHz. Finally, in Figures 3.c and 4.c, simulated radiation patterns of the antenna are shown for $\mathrm{H}$ plane (phi $=90$ ), for $2.337 \mathrm{GHz}$ and 5.6 GHz. In H-plane, the antenna has a co/cross pol. ratio of $20 \mathrm{~dB}$ at $2.337 \mathrm{GHz}$ and almost $45 \mathrm{~dB}$ at $5.6 \mathrm{GHz}$. The lower co/cross pol. ratio for 2.337 $\mathrm{GHz}$ may be caused by the radiation from the quarter wave short circuited stubs which are used for RF pin diodes biasing. The calculated gain of the antenna from the radiation pattern is 7.26 $\mathrm{dBi}$ at $2.337 \mathrm{GHz}$ and $6.93 \mathrm{dBi}$ at $5.6 \mathrm{GHz}$.

\section{Conclusion and Future Work}

A reconfigurable microstrip patch antenna with $\mathrm{RF}$ pin diode switches are presented for dual band of $2.4 \mathrm{GHz}$ and 5.6 GHz SDR applications. Band switching is simply obtained by biasing the four RF pin diodes. The antenna is well matched and achieves a gain of approximately $7 \mathrm{~dB}$ at both WLAN bands with similar radiation patterns. Simulation results and early measurement results will be presented at the conference.

\section{References:}

[1] J.T. Aberle et. al., "Automatically tuning antenna for software-defined and cognitive radio", Proceedings of Software Defined Radio 2005 conference, Orange County, CA, November 2005.

[2] J. Liang and H.Y.D. Yang, '’Frequency reconfigurable printed inverted-F antennas', 2008 IEEE APS/URSI conference proceedings, San Diego, July 2008.

[3] J.A. DeSignor and J. Venkataraman, 'Reconfigurable dual frequency microstrip patch antenna asing RF MEMS switches", Proceedings of ACES 2008, Niagara Falls, Canada, March 2008.

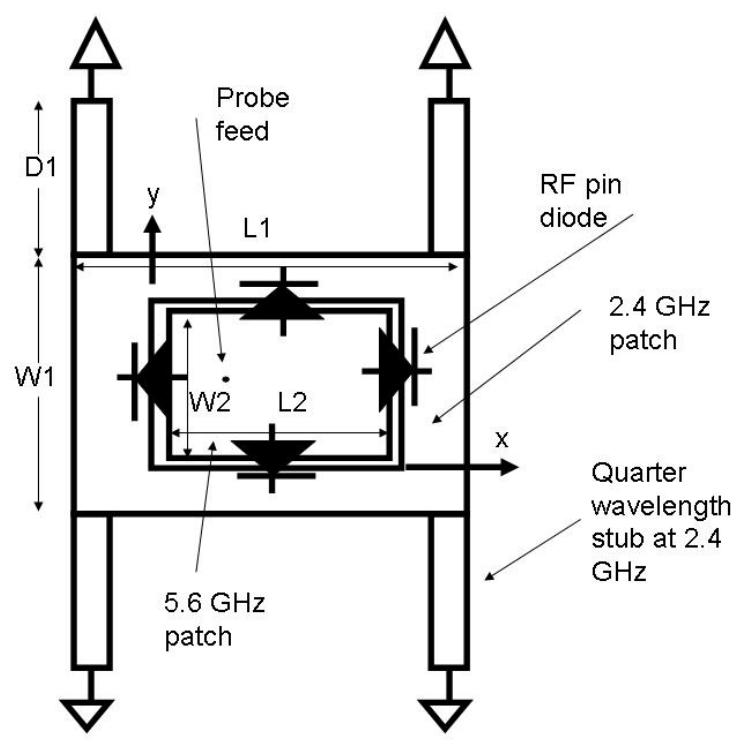

Fig. 1 Dual band 2.4/5.6 GHz reconfigurable patch antenna

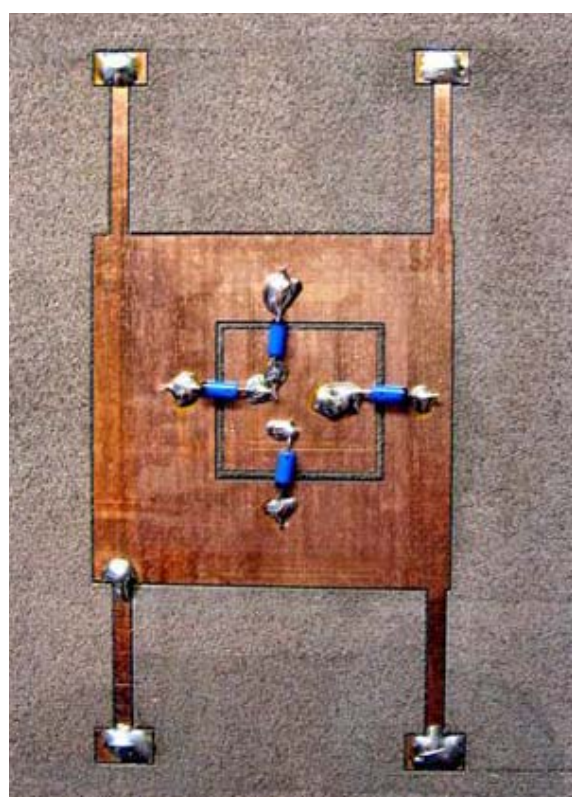

Fig. 2 Fabricated reconfigurable patch antenna 


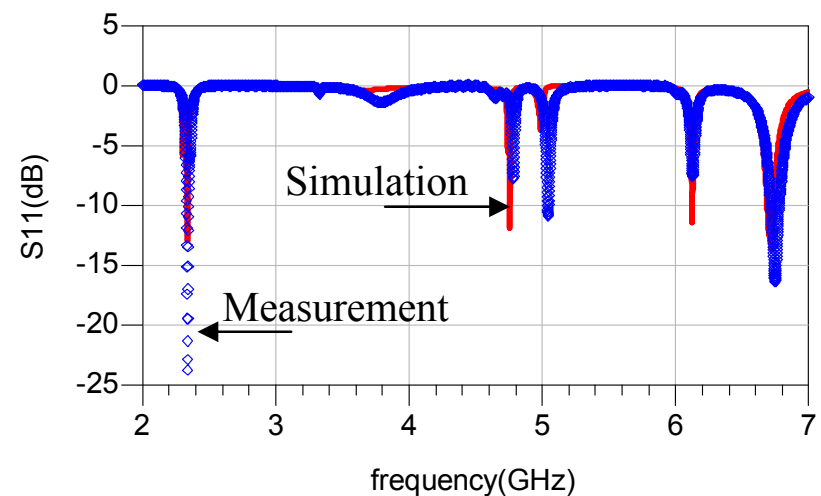

(a)

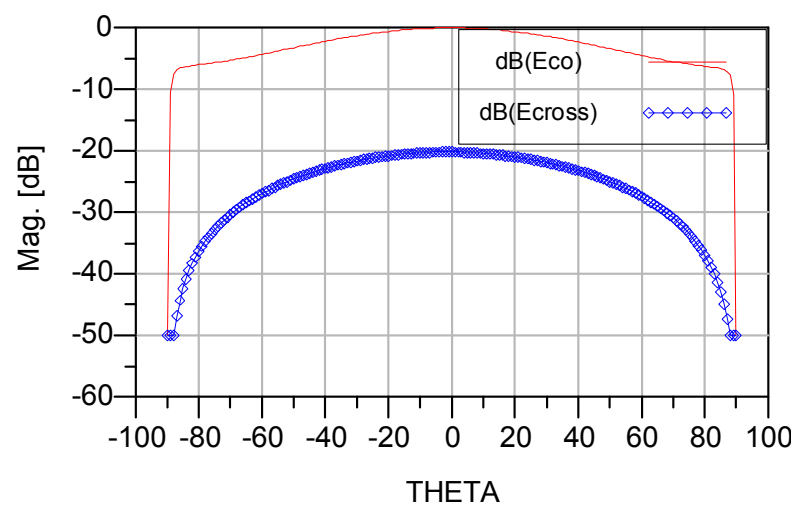

(b)

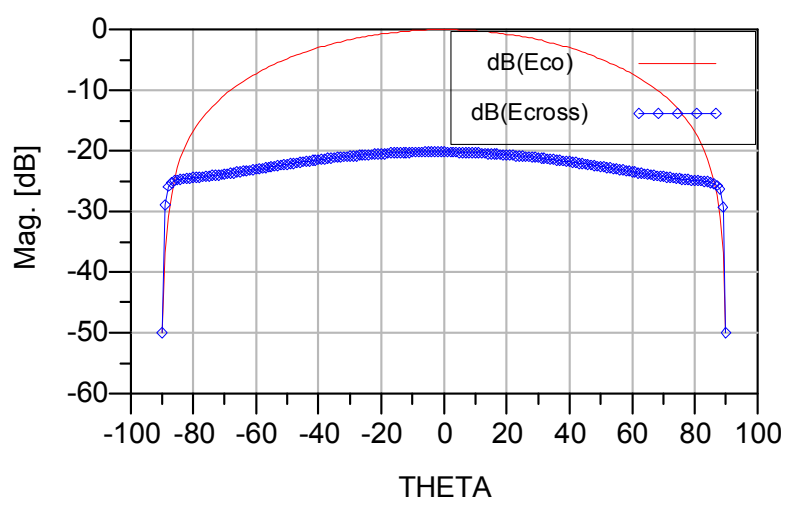

(c)

Fig. 3 Simulated and measured a) input impedance of the antenna versus frequency $(\mathrm{GHz})$

-all RF pin diodes are on.

b) simulated radiation pattern of the reconfigurable antenna

E-plane (phi=0) at $2.337 \mathrm{GHz}$

Eco $=$ Exz, Ecross $=$ Eyz

c) simulated radiation pattern of the antenna on

H-plane (phi=90) at $2.337 \mathrm{GHz}$

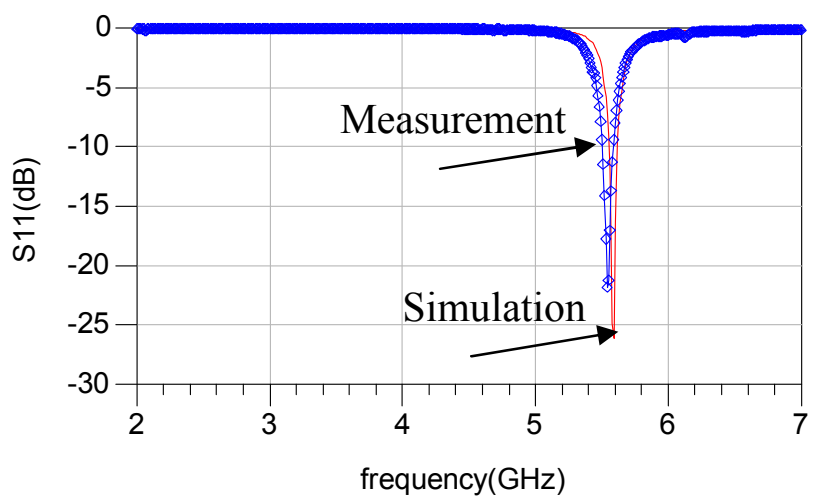

(a)

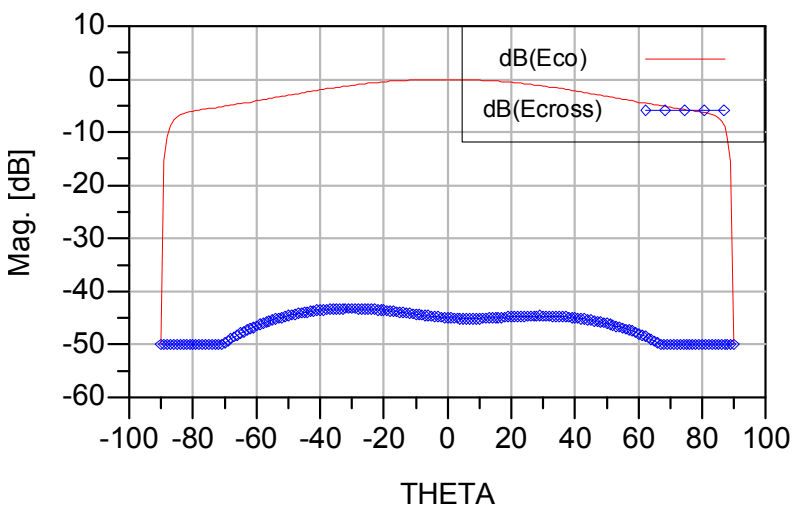

(b)

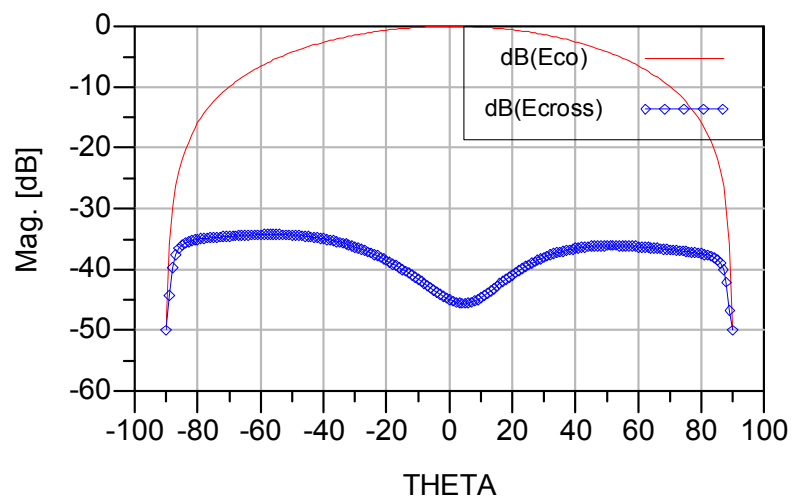

(c)

Fig. 4 Simulated and measured a) input impedance of the antenna versus frequency $(\mathrm{GHz})$

-all RF pin diodes are off.

b) simulated radiation pattern of the reconfigurable antenna

E-plane (phi $=0)$ at $5.6 \mathrm{GHz}$

Eco $=$ Exz, Ecross $=$ Eyz

c) simuated radiation pattern of the antenna on

H-plane $($ phi $=90)$ at $5.6 \mathrm{GHz}$ 